\title{
Cost Effectiveness of First-Line Oral Therapies for Pulmonary Arterial Hypertension: A Modelling Study
}

\author{
Kathryn Coyle ${ }^{1} \cdot$ Doug Coyle ${ }^{1,2} \cdot$ Julie Blouin $^{3} \cdot$ Karen Lee $^{3} \cdot$ Mohammed F. Jabr $^{3}$. \\ ${\text { Khai } \operatorname{Tran}^{3} \cdot \text { Lisa Mielniczuk }^{4} \cdot \text { John Swiston }}^{5} \cdot$ Mike Innes $^{3}$
}

Published online: 6 January 2016

(c) The Author(s) 2016. This article is published with open access at Springerlink.com

\begin{abstract}
Background In recent years, a significant number of costly oral therapies have become available for the treatment of pulmonary arterial hypertension (PAH). Funding decisions for these therapies requires weighing up their effectiveness and costs.

Objective The aim of this study was to assess the cost effectiveness of monotherapy with oral $\mathrm{PAH}$-specific therapies versus supportive care as initial therapy for patients with functional class (FC) II and III PAH in Canada.

Methods A cost-utility analysis, from the perspective of a healthcare system and based on a Markov model, was designed to estimate the costs and quality-adjusted lifeyears (QALYs) associated with bosentan, ambrisentan, riociguat, tadalafil, sildenafil and supportive care for $\mathrm{PAH}$ in treatment-naïve patients. Separate analyses were conducted for cohorts of patients commencing therapy at FC II and III PAH. Transition probabilities, based on the relative risk of improving and worsening in FC with treatment versus placebo, were derived from a recent network meta-
\end{abstract}

Electronic supplementary material The online version of this article (doi:10.1007/s40273-015-0366-8) contains supplementary material, which is available to authorized users.

Kathryn Coyle

Kathryn.Coyle@brunel.ac.uk

1 Brunel University, Gaskell building, Uxbridge, Middlesex UB8 3PH, UK

2 University of Ottawa, Ottawa, ON, Canada

3 Canadian Agency for Drugs and Technologies in Health (CADTH), Ottawa, ON, Canada

4 University of Ottawa Heart Institute, Ottawa, ON, Canada

5 University of British Columbia, Vancouver, BC, Canada analysis. Utility values and costs were obtained from published data and clinical expert opinion. Extensive sensitivity analyses were conducted.

Results Analysis suggests that sildenafil is the most costeffective therapy for PAH in patients with FC II or III. Sildenafil was both the least costly and most effective therapy, thereby dominating all other treatments. Tadalafil was also less costly and more effective than supportive care in FC II and III; however, sildenafil was dominant over tadalafil. Even given the uncertainty within the clinical inputs, the probabilistic sensitivity analysis showed that apart from sildenafil and tadalafil, the other PAH therapies had negligible probability of being the most cost effective. Conclusion The results show that initiation of therapy with sildenafil is likely the most cost-effective strategy in PAH patients with either FC II or III disease.

\section{Key Points for Decision Makers}

The results indicate that initiation of therapy with sildenafil in patients with functional class (FC) II and III PAH would result in probable cost savings compared with riociguat, bosentan, ambrisentan $5 \mathrm{mg}$, ambrisentan $10 \mathrm{mg}$ and tadalafil.

The study findings do not support differential funding of PAH therapies for patients with FC II versus FC III disease based on current evidence.

\section{Introduction}

Pulmonary arterial hypertension (PAH) is an uncommon progressive condition characterised by increased pulmonary vascular resistance which often leads to right 
ventricular heart failure and death [1]. Historically, most cases of the disease were diagnosed in young adulthood, with women being preferentially affected relative to men [2]; however, more recently the median age at which people are diagnosed has increased to between 50 and 60 years [3]. PAH not only shortens affected individual's lives but also significantly impacts their quality of life, limiting their ability to work and perform day-to-day activities, often leading to social isolation [4].

The prevalence of PAH within Europe has been reported as between 15 and 50 cases per million population [5]. Given that Canada does not have a PAH registry, assuming a similar percentage of the population is affected within Canada would result in an estimated 500 and 1800 cases.

If left untreated, the prognosis for patients with PAH is poor. In historical registries, such as one dating from 1981, the median survival was only 2.8 years [2]; however, based on a more recent registry, the median survival was estimated at 7 years [6]. Although survival appears to be greater in more recent times, the factors that have contributed to this difference are unclear. Significant changes have occurred related to both the diagnosis of PAH and the classification of the disease over this timeframe. The management has also changed, with more awareness of the disease, a greater number of patients treated within specialist centers, and increased therapeutic options.

Given the significant morbidity and mortality associated with the disease, research into new therapies has been focused on both improving patient's quality of life, through reducing symptoms, and increasing functioning and extending survival.

Epoprostenol was the first PAH-specific therapy available and has been shown to improve patient outcomes, including symptoms, hemodynamics, and survival [7]. However, epoprostenol is burdensome to administer as it necessitates a central venous catheter (CVC) and must be stored under refrigeration. In recent years, several oral therapies for PAH have become available that are much less onerous to administer, leading to epoprostenol often being reserved for second- or third-line therapy [5]. Unlike epoprostenol, the oral agents generally do not require hospitalization for initiation and avoid the potentially serious complications associated with a CVC. There are also potential cost advantages to oral therapies as the cost of the medication is generally lower.

Oral therapies for PAH fall into one of three categories, namely endothelial receptor antagonists, which include ambrisentan, bosentan and macitentan; phosphodiesterase type-5 inhibitors, specifically sildenafil and tadalafil; and the most recently available agent riociguat, a soluble guanylate cyclase stimulator.

The choice of oral therapy for initiation of treatment is challenging as there are very few head-to-head clinical trials from which to assess the comparative effectiveness of these agents. However, recent developments in network metaanalysis techniques have provided a method by which the comparative effectiveness of treatments can be estimated in the absence of direct head-to-head trials [8]. One such recent analysis compared the effectiveness of available PAH treatments in patients who are naïve to $\mathrm{PAH}$ therapies and therefore may be helpful in guiding the choice of agent for initiation of therapy [9]. Given the current climate of restricted budgets for healthcare treatments, the choice of therapeutic agent should be informed not only by the comparative clinical effectiveness of treatments but also the comparative cost effectiveness, thereby ensuring maximum efficiency of the healthcare system.

We conducted an economic analysis to address the decision problem relating to what is the optimal therapy to initiate treatment for PAH patients. Analysis compared the cost effectiveness of oral PAH treatments with supportive care alone as initial therapy for patients with New York Heart Association functional class (FC) II and III disease in Canada. The estimated efficacy of the oral PAH therapies was derived from the recent network meta-analysis.

\section{Methods}

\subsection{Analytical Framework}

We developed a Markov model within Microsoft Excel (Microsoft Corporation, Remond, WA, USA) to simulate the long-term outcomes with treatment in the absence of long-term clinical trial data. The model consisted of seven states-NYHA FC I, II, III and IV with oral therapy alone, and FC III and IV with oral therapy in combination with epoprostenol and death (Fig. 1). With every 3-month cycle, transition probabilities for the improvement in FC, worsening in FC, and death determined the proportion of individuals who transition to different states. A 3-month cycle length was chosen as it closely approximates the time point at which effectiveness of PAH therapies was assessed within the clinical trials. A proportion of patients deteriorating to FC IV are assumed to initiate epoprostenol therapy, which was estimated at $50 \%$ within the base-case analysis, based on expert clinical opinion (JS/LM). With initiation of epoprostenol, patients may improve to FC III. Death is an absorbing state. We estimated the impact of initial treatments for PAH through their effects on qualityadjusted life-years (QALYs) and costs over a lifetime, and used these values to calculate incremental cost-effectiveness ratios (ICERs) for a comparative cost-effectiveness analysis. Analysis was conducted from the perspective of the Ontario healthcare system [10]. The time horizon for the base analysis was lifetime (30 years or death), with 
Fig. 1 Model schematic. FC New York Heart Association functional class, $P A H$ pulmonary arterial hypertension, $S C$ supportive care, epo epoprostenol

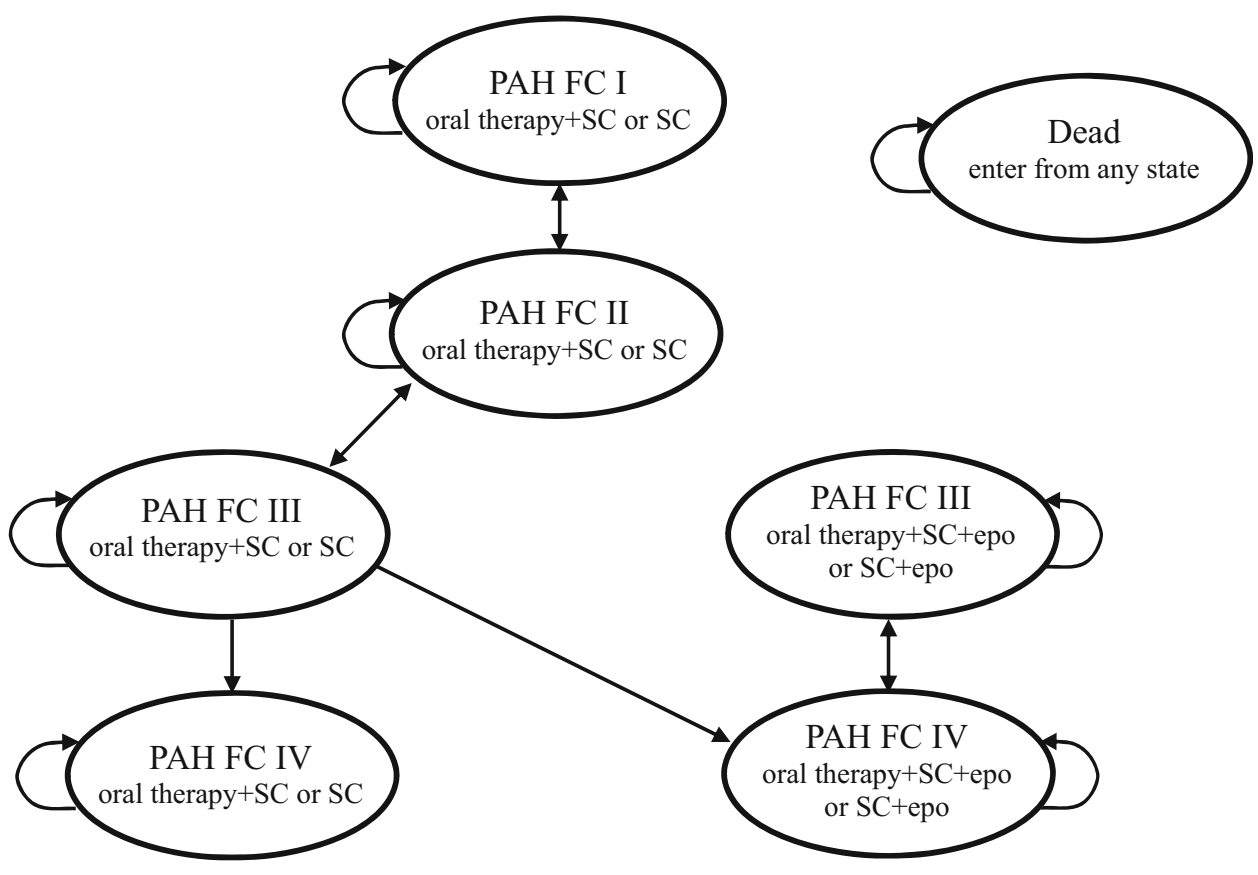

future costs and outcomes discounted at a rate of $5 \%$ per annum [10].

The clinical experts were selected based on their expertise in the treatment of pulmonary hypertension in Canada, and consensus was reached through negotiation with alternative values tested within sensitivity analyses.

\subsection{Patient Population}

Analysis was conducted for two separate patient cohortsthose initiating therapy in PAH FC II, and those initiating therapy in PAH FC III. For both cohorts, it was assumed that treatment is initiated at 50 years of age and $70 \%$ of the cohort is female, based on a PAH incidence registry [11].

\subsection{Comparators}

Comparators were riociguat, bosentan, ambrisentan 5 and $10 \mathrm{mg}$, sildenafil and tadalafil in combination with supportive care compared with supportive care alone. Macitentan was not included as there were no clinical data available specific to patients who are naïve to $\mathrm{PAH}$ therapy.

\subsection{Model Variables}

Data were required relating to disease progression, mortality by FC, the relative impact of treatment on disease progression, and adverse event rates associated with treatment, in addition to utility values and costs for the relevant health states within the model (Table 1).

\subsubsection{Baseline Disease Progression}

Patients receiving only supportive care could either improve, by moving to a milder FC, remain within the same FC, or their disease could worsen, represented by a move to the next more severe FC. These rates were derived from the placebo arms of the included clinical trials, as estimated within the network meta-analysis.

\subsubsection{Mortality}

We derived age- and sex-specific annual mortality rates from Canadian life tables [12]. To account for the impact of the disease on mortality, we estimated the relative rate of death with progression to FC II, III, and IV from a recent PAH registry [13]. The registry, which evaluated 578 PAH patients between 1982 and 2006, found an increased rate of death compared with FC I disease, with a hazard ratio of $4.51 \quad(95 \%$ CI 1.37-14.84) in FC II, 7.94 (95\% CI 2.53-24.97) in FC III, and 11.6 (95\% CI 3.68-36.63) in FC IV. We applied the increased risk of death with $\mathrm{PAH}$ to the underlying age- and sex-specific mortality rates, and calibrated the model to reflect the PAH registry overall survival [14]. We also validated the survival predictions 
Table 1 Transition probabilities, mortality rates, relative effects, costs and utilities by functional class and treatment

\begin{tabular}{|c|c|c|}
\hline \multicolumn{3}{|l|}{ Costs } \\
\hline Resource & Value & Reference \\
\hline \multicolumn{3}{|l|}{ Monthly cost of PAH medications } \\
\hline Ambrisentan $5 \mathrm{mg}$ or $10 \mathrm{mg}$ once daily & $\$ 4028$ & \multirow[t]{6}{*}[26]{} \\
\hline Bosentan $62.5-125 \mathrm{mg}$ bid & $\$ 4219$ & \\
\hline Sildenafil $20 \mathrm{mg}$ tid & $\$ 1099$ & \\
\hline Tadalafil $40 \mathrm{mg}$ once daily & $\$ 881$ & \\
\hline Riociguat $1-2.5 \mathrm{mg}$ tid & $\$ 4216$ & \\
\hline Epoprostenol (initiation: 2-27 ng/kg/min; subsequent: $27-50 \mathrm{ng} / \mathrm{kg} / \mathrm{min}$ ) & First 3 months: $\$ 1,758$; subsequent months: $\$ 3,749$ & \\
\hline \multicolumn{3}{|l|}{ Average monitoring costs, per month (SE) } \\
\hline Ambrisentan $^{\mathrm{a}}$ & $\$ 16(4.1)$ & \multirow[t]{2}{*}[30]{} \\
\hline Bosentan $^{\mathrm{b}}$ & $\$ 26(6.6)$ & \\
\hline \multicolumn{3}{|l|}{ Epoprostenol specific costs (SE) } \\
\hline Initiation costs ${ }^{\mathrm{c}}$ & $\$ 9759$ (2439.7) & {$[28,29]$} \\
\hline Infusion supply costs per day & $\$ 53(13.25)$ & {$[25]$} \\
\hline Cost to treat an episode of sepsis & $\$ 20,966(5241)$ & [31] \\
\hline Cost for replacement of CVC (every 2 years and due to infection) & $\$ 166(41.5)$ & {$[29]$} \\
\hline \multicolumn{3}{|l|}{ Average cost of supportive care medications, per month ${ }^{\mathrm{d}}(\mathrm{SE})$} \\
\hline Functional class II & $\$ 30(7.5)$ & \multirow[t]{4}{*}[44,45]{} \\
\hline Functional class III & $\$ 116(28.9)$ & \\
\hline Functional class IV & $\$ 287(71.7)$ & \\
\hline Functional class IV supportive care arm & $\$ 400(99.9)$ & \\
\hline \multicolumn{3}{|l|}{ Cost of continuing PAH care, per month ${ }^{\mathrm{e}}$ (SE) } \\
\hline Functional class II & $\$ 228(57.0)$ & \multirow[t]{3}{*}[20,29,46]{} \\
\hline Functional class III & $\$ 727(181.9)$ & \\
\hline Functional class IV & $\$ 2267(566.7)$ & \\
\hline \multicolumn{3}{|l|}{ Transition probabilities } \\
\hline \multicolumn{3}{|l|}{ Supportive care } \\
\hline Probability of FC improvement & 0.10 & \multirow[t]{2}{*}{ [9] } \\
\hline Probability of FC worsening & 0.12 & \\
\hline \multicolumn{3}{|l|}{ Relative risk of FC improvement versus supportive care $(95 \% \mathrm{CrI})$} \\
\hline Ambrisentan $5 \mathrm{mg}$ & $1.06(0.61,1.79)$ & \multirow[t]{7}{*}[9]{$^{\mathrm{f}}$} \\
\hline Ambrisentan $10 \mathrm{mg}$ & $1.21(0.62,2.23)$ & \\
\hline Bosentan & $2.05(1.25,3.32)$ & \\
\hline Sildenafil & $3.71(1.76,7.29)$ & \\
\hline Tadalafil & $2.67(1.11,5.76)$ & \\
\hline Riociguat & $0.98(0.45,2.08)$ & \\
\hline Epoprostenol & $9.42(5.65,17.48)$ & \\
\hline \multicolumn{3}{|l|}{ Relative risk of functional class worsening versus supportive care $(95 \% \mathrm{CrI})$} \\
\hline Ambrisentan $5 \mathrm{mg}$ & $0.11(0.03,0.34)$ & \multirow[t]{7}{*}[9]{$^{\mathrm{g}}$} \\
\hline Ambrisentan $10 \mathrm{mg}$ & $0.25(0.05,0.81)$ & \\
\hline Bosentan & $0.46(0.18,1.04)$ & \\
\hline Sildenafil & $0.27(0.04,1.10)$ & \\
\hline Tadalafil & $0.45(0.11,1.44)$ & \\
\hline Riociguat & $0.22(0.07,0.63)$ & \\
\hline Epoprostenol & $0.40(0.15,0.93)$ & \\
\hline \multicolumn{3}{|l|}{ Mortality rates } \\
\hline \multicolumn{3}{|l|}{ Relative risk of mortality versus FC I } \\
\hline Functional class II vs. FC I & $4.51(1.37,14.84)$ & \multirow[t]{3}{*}[13]{} \\
\hline Functional class III vs. FC I & $7.94(2.53,24.97)$ & \\
\hline Functional class IV vs. FC I & $11.60(3.68,36.63)$ & \\
\hline Increased risk of mortality with sepsis (per person month) & 0.000678 & [7] \\
\hline
\end{tabular}


Table 1 continued

\begin{tabular}{lll}
\hline Costs & & \\
\hline Resource & Value & Reference \\
\hline Utilities & & \\
Functional class I & $0.73(0.64,0.82)$ & \\
Functional class II & $0.67(0.57,0.77)$ & \\
Functional class III & $0.60(0.50,0.70)$ & \\
Functional class IV & $0.52(0.43,0.61)$ & 0.108 \\
Disutility with sepsis, over 3 months & {$[24]$} \\
\hline
\end{tabular}

CrI credible interval, $P A H$ pulmonary arterial hypertension, bid twice daily, tid three times daily, $C V C$ central venous catheter, $S E$ standard error, $N Y H A$ New York Heart Association, $F C$ functional class

All costs are expressed in Canadian dollars

${ }^{a}$ Monthly liver function tests and annual pregnancy test with ambrisentan

${ }^{\mathrm{b}}$ Monthly liver function tests and monthly pregnancy tests with bosentan

${ }^{c}$ Assumed in $50 \%$ of patients, epoprostenol is initiated within the hospital, and for $50 \%$ through day surgery. Also includes training and CVC insertion costs

${ }^{\mathrm{d}}$ Warfarin $5 \mathrm{mg}$ daily in $53 \%$ of patients, furosemide $100 \mathrm{mg}$ daily in $69 \%$ of patients, digoxin $0.125 \mathrm{mg}$ daily in $26 \%$ of patients, and home oxygen therapy in $5 \%$ of patients with NYHA FC II, $27 \%$ of patients with NYHA FC III and $71 \%$ of patients with NYHA FC IV receiving PAH-specific therapies, and $100 \%$ of patients with NYHA FC IV receiving supportive care

e Includes general practitioner visits, specialist visits, nurse visits, hospitalizations, emergency room visits, and therapeutic procedures (echocardiograph and blood work)

${ }^{\mathrm{f}}$ Fixed effect model, naïve population network meta-analysis, Table 184, Appendix 11

${ }^{\mathrm{g}}$ Fixed effect model, naïve population network meta-analysis, Table 188, Appendix 11

of the model against a second PAH registry [15] (see electronic supplementary Appendix).

\subsubsection{Efficacy of Treatment}

We derived the transition probabilities for each of the medications under study by applying the relative effects of therapy from a recent network meta-analysis, which estimated the comparative impact of PAH therapies on the improvement and worsening in $\mathrm{FC}$, to the baseline probabilities for FC improvement and worsening with supportive care alone [9]. Those who did not improve or worsen remained within their current $\mathrm{FC}$ for the cycle.

In the absence of long-term clinical data, assumptions were required regarding the persistence of the impact of the medications. Clinical trials generally measured changes in FC at 12-16 weeks; therefore, the PAH medications were assumed to result in improvements in FC during the first cycle of the model. Clinical trial evidence also supports that these medications reduce the risk of FC worsening [16-19]. Although the duration of this effect has not been well-documented within long-term controlled trials, within the base-case analysis, we assumed that it persisted throughout the lifetime of the patient. These assumptions were based on expert clinical opinion (JS/LM), are in line with other cost-effectiveness analyses, and were tested within sensitivity analyses [20].

Studies have found conflicting results with respect to the effect of treatment on survival. A meta-analysis from 2009 reported a $43 \%$ reduction in mortality with PAH- targeted treatments, whereas a systematic review of the literature from 2007 found little evidence for a survival benefit with PAH-targeted treatments [21, 22]. With this model, we assumed that treatments had no independent effect on mortality but rather affected mortality through delaying the progression of the disease. This avoided double counting any impact of the medications on survival.

\subsubsection{Adverse Events}

Adverse events that have a clear impact on utility and costs, specifically those that were considered serious, were included within the analysis. Adverse events for oral PAH therapies were not included as they were generally minor and were comparable to those with placebo or supportive care. In patients receiving epoprostenol there is an increased risk of infections associated with the presence of a CVC and the potential for the severe complication of sepsis, which is associated with increased costs, a utility decrement, and increased mortality. Within the model, we included the costs for CVC infections, as well as the costs, disutility, and increased mortality associated with sepsis, in patients receiving epoprostenol.

\subsubsection{Utility Values}

Although many clinical trials of PAH therapies report having measured quality of life, none have reported the impact of treatment in a format that would allow estimation 
of QALYs. We therefore assigned utility values based on the severity of PAH, as measured by FC. These were obtained from a published study deriving utility values using the SF-36 with 177 Australian PAH patients [23]. Within this study, the utility value was shown to decrease with increasing severity of PAH, as measured by a worsening FC.

In addition, for those developing sepsis while on epoprostenol, a utility decrement of 0.108 was applied for the 3 -month cycle in which the event occurred. This corresponds to an annual utility decrement of 0.027 [24].

\subsubsection{Cost Variables}

The costs associated with initiation of therapy, ongoing treatment, and monitoring the disease were also included. The costs related to the diagnosis of PAH were not included within the model as we assumed they would be incurred by all PAH patients, regardless of the initial treatment strategy, and therefore would not impact the relative cost effectiveness.

According to expert clinical opinion (JS/LM), oral therapies are generally initiated in the patient's home and therefore do not incur any costs for initiation; however, a portion of patients are typically hospitalised during initiation of epoprostenol. We therefore included the hospitalization and healthcare professional costs for initiation of epoprostenol.

For the cost of PAH therapies, we included both the medication costs, with mark-up and dispensing fees, and the cost of administration supplies for epoprostenol. Medication and diluent costs were obtained from a provincial drug formulary, and the cost of equipment was derived from a previous Canadian analysis [25, 26].

With both the supportive care strategy and each of the PAH treatment strategies, the costs of non-PAH-specific therapies, including warfarin in $53 \%$ of patients, digoxin in $26 \%$ of patients and furosemide in $69 \%$ of patients, were also incorporated [27]. Oxygen use was assumed to vary based on FC, with $5 \%$ of patients receiving it in FC II, $27 \%$ in FC III, and $71 \%$ in FC IV for those receiving PAH treatment strategies, and $100 \%$ in FC IV in those in the supportive care strategy [20].

The costs of supportive care for patients diagnosed with PAH vary by FC. A recent UK study reported resource use by patients with FC II, III, and IV PAH under the categories of hospitalizations, specialist and non-specialist clinic visits, nurse visits, and emergency room visits [20]. We applied current Canadian costs to this resource data to estimate the average ongoing cost by FC [28, 29]. Within the base-case analysis, in the absence of conflicting data, we assumed that there were no ongoing costs for patients within FC I. This assumption was tested in sensitivity analyses. Patients were also assumed to undergo a standard set of PAH monitoring tests (renal function tests, blood tests, echocardiogram) for which current Canadian costs were applied $[29,30]$. For each patient developing sepsis, a treatment cost of Can $\$ 19,457$ was incorporated [31]. For CVC infections, it was assumed that the catheter would be replaced both when the infection occurred and every 2 years in the absence of an infection, at a cost of Can $\$ 166$ [29].

Costs were reported in 2013 Canadian dollars with any inflation adjustments based on the Bank of Canada Inflation Calculator [32].

\subsection{Sensitivity Analysis}

Deterministic sensitivity analyses were conducted to assess the impact of parameter uncertainty and structural uncertainty on the cost-effectiveness outcome. Alternative assumptions regarding the persistence of the impact of treatment on FC worsening, an alternative source of utility values [33], a generic price for bosentan and epoprostenol, and varying the epoprostenol dose to the extremes of the prescribed dosage range were tested. Structural uncertainty was examined through producing undiscounted estimates of cost and QALYs, reducing the time horizon to 10 years, and by assuming either no patients or all patients initiated epoprostenol therapy upon deterioration to FC IV.

A probabilistic sensitivity analysis with 5000 replications was conducted in which each model input parameter was represented by a probability distribution [34]. Standard distributions were used for each input parameter: log normal distribution for odds ratios and relative risks, gamma distributions for costs, and beta distributions for utilities. As the source documents for the costs did not contain estimates of uncertainty, we estimated a standard error at $25 \%$ of the mean [34].

\section{Results}

The results of the cost-effectiveness analysis were similar for patients initiating therapy with both FC II PAH and FC III (Table 2). In both cases, sildenafil was the least costly strategy and produced the greatest QALYs, thereby dominating all other therapies (Fig. 2). The increase in QALYs is primarily due to the impact of sildenafil in delaying disease progression as it produced a greater effect on FC improvement than all other treatments. The lower costs can be attributed primarily to the lower acquisition cost of sildenafil, which is approximately one-third of that of other PAH therapies (Table 3).

Extensive sensitivity analyses found the results to be robust to changes in most model assumptions. Sildenafil 
Table 2 Results by PAH functional class at initiation of therapy

\begin{tabular}{|c|c|c|c|}
\hline Treatment & Discounted costs $(\$)$ & Discounted QALYs & $\begin{array}{l}\text { Incremental cost per } \\
\text { QALY compared with sildenafil }\end{array}$ \\
\hline \multicolumn{4}{|l|}{ Functional class II } \\
\hline Sildenafil & $\$ 146,254$ & 4.663 & \\
\hline Tadalafil & $\$ 153,245$ & 4.002 & Dominated by sildenafil \\
\hline Supportive care & $\$ 155,156$ & 3.218 & Dominated by sildenafil \\
\hline Ambrisentan $5 \mathrm{mg}$ & $\$ 377,187$ & 4.634 & Dominated by sildenafil \\
\hline Ambrisentan $10 \mathrm{mg}$ & $\$ 377,523$ & 4.217 & Dominated by sildenafil \\
\hline Riociguat & $\$ 388,491$ & 4.244 & Dominated by sildenafil \\
\hline Bosentan & $\$ 406,282$ & 3.904 & Dominated by sildenafil \\
\hline \multicolumn{4}{|l|}{ Functional class III } \\
\hline Sildenafil & $\$ 181,119$ & 3.284 & \\
\hline Tadalafil & $\$ 200,584$ & 3.013 & Dominated by sildenafil \\
\hline Supportive care & $\$ 204,285$ & 2.687 & Dominated by sildenafil \\
\hline Ambrisentan $5 \mathrm{mg}$ & $\$ 351,573$ & 3.180 & Dominated by sildenafil \\
\hline Ambrisentan $10 \mathrm{mg}$ & $\$ 376,884$ & 3.043 & Dominated by sildenafil \\
\hline Riociguat & $\$ 383,582$ & 3.045 & Dominated by sildenafil \\
\hline Bosentan & $\$ 412,979$ & 2.960 & Dominated by sildenafil \\
\hline
\end{tabular}

All costs are expressed in Canadian dollars

Dominated more costly and fewer QALYs, $Q A L Y$ quality-adjusted life-year, $P A H$ pulmonary arterial hypertension

Fig. 2 Incremental costs versus QALYs for oral PAH therapies versus supportive care in $\mathrm{PAH}$ functional class III. $Q A L Y S$ quality-adjusted life-years, $P A H$ pulmonary arterial hypertension

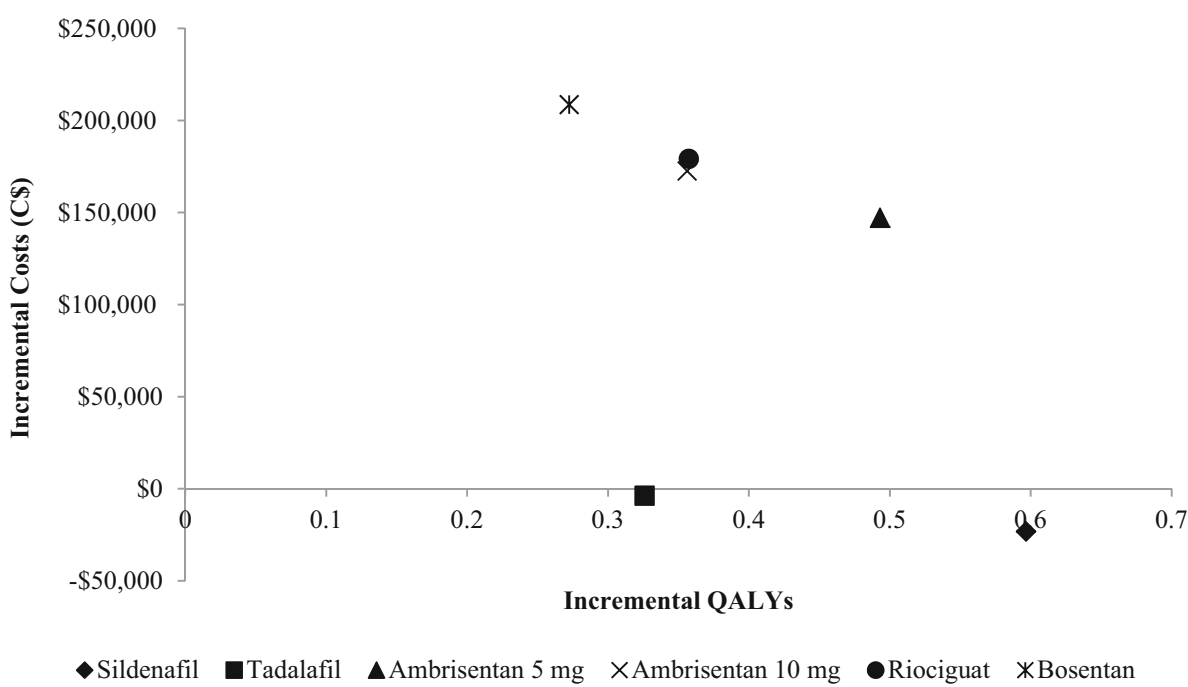

remained the dominant treatment in all sensitivity analyses, except when alternative assumptions regarding the percentage of patients initiating epoprostenol and the waning of the treatment effect were incorporated. If epoprostenol is not introduced upon deterioration to FC IV in any treatment strategy, the ICER increases to Can $\$ 29,035$ per QALY for sildenafil versus supportive care in FC II, and to Can\$45,349 per QALY in FC III.

When the treatment effect was assumed to wane at various rates after the first 18 months of therapy, reaching that of supportive care after either a further 10 years or a further 5 years in some cases, sildenafil no longer dominated supportive care. However, sildenafil remained the most cost-effective treatment option, with an ICER of less than Can $\$ 35,000$ per QALY versus supportive care in both waning scenarios, in patients with FC II and III disease. Full details of the sensitivity analysis are provided in the electronic supplementary Appendix.

In the probabilistic sensitivity analysis, sildenafil was the most cost-effective therapy in the majority of 
Table 3 Breakdown of discounted lifetime costs by treatment at initiation

\begin{tabular}{|c|c|c|c|c|c|c|c|c|}
\hline $\begin{array}{l}\text { Functional } \\
\text { class }\end{array}$ & Item & $\begin{array}{l}\text { Ambrisentan } \\
5 \mathrm{mg}\end{array}$ & $\begin{array}{l}\text { Ambrisentan } \\
10 \mathrm{mg}\end{array}$ & Bosentan & Sildenafil & Tadalafil & Riociguat & $\begin{array}{l}\text { Supportive } \\
\text { care }\end{array}$ \\
\hline \multirow[t]{5}{*}{ II } & PAH-specific drugs & $\$ 341,088$ & $\$ 317,768$ & $\$ 314,817$ & $\$ 93,602$ & $\$ 66,920$ & $\$ 334,140$ & $\$ 0$ \\
\hline & Monitoring/therapeutic procedures ${ }^{\mathrm{a}}$ & $\$ 3131$ & $\$ 2913$ & $\$ 3484$ & $\$ 1768$ & $\$ 1573$ & $\$ 1643$ & $\$ 1394$ \\
\hline & Hospital/ER/clinic visits ${ }^{\mathrm{b}}$ & $\$ 23,882$ & $\$ 32,953$ & $\$ 42,901$ & $\$ 29,213$ & $\$ 41,522$ & $\$ 31,638$ & $\$ 63,053$ \\
\hline & Supportive care drugs ${ }^{c}$ & $\$ 3608$ & $\$ 4886$ & $\$ 6182$ & $\$ 4332$ & $\$ 5991$ & $\$ 4711$ & $\$ 10,833$ \\
\hline & Epoprostenol $^{\mathrm{d}}$ & $\$ 5478$ & $\$ 19,002$ & $\$ 38,899$ & $\$ 17,339$ & $\$ 37,239$ & $\$ 16,358$ & $\$ 79,921$ \\
\hline \multirow[t]{5}{*}{ III } & PAH-specific drugs & $\$ 261,295$ & $\$ 253,884$ & $\$ 261,209$ & $\$ 72,780$ & $\$ 55,154$ & $\$ 265,827$ & $\$ 0$ \\
\hline & Monitoring/therapeutic procedures ${ }^{\mathrm{a}}$ & $\$ 2385$ & $\$ 2316$ & $\$ 2878$ & $\$ 1367$ & $\$ 1291$ & $\$ 1300$ & $\$ 1200$ \\
\hline & Hospital/ER/clinic visits ${ }^{\mathrm{b}}$ & $\$ 51,793$ & $\$ 59,704$ & $\$ 66,052$ & $\$ 53,580$ & $\$ 64,352$ & $\$ 58,926$ & $\$ 79,770$ \\
\hline & Supportive care drugs ${ }^{c}$ & $\$ 8052$ & $\$ 8883$ & $\$ 9500$ & $\$ 7971$ & $\$ 9720$ & $\$ 8825$ & $\$ 13,986$ \\
\hline & Epoprostenol $^{\mathrm{d}}$ & $\$ 28,049$ & $\$ 52,098$ & $\$ 73,341$ & $\$ 45,421$ & $\$ 70,518$ & $\$ 48,703$ & $\$ 109,328$ \\
\hline
\end{tabular}

$E R$ emergency room, $P A H$ pulmonary arterial hypertension, $N Y H A$ New York Heart Association $F C$ functional class

All costs are expressed in Canadian dollars

a Includes monthly liver function tests for bosentan and ambrisentan, monthly pregnancy test for bosentan, and annual pregnancy tests for ambrisentan; echocardiograms, renal function, and blood work for all therapies

b Includes general practitioner visits, specialist visits, nurse visits, hospitalizations, emergency room visits, therapeutic procedures (echocardiograph and blood work)

${ }^{\mathrm{c}}$ Warfarin $5 \mathrm{mg}$ daily in $53 \%$ of patients, furosemide $100 \mathrm{mg}$ daily in $69 \%$ of patients, digoxin $0.125 \mathrm{mg}$ daily in $26 \%$ of patients, and home oxygen therapy in $5 \%$ of patients with NYHA FC II, $27 \%$ of patients with NYHA FC III and $71 \%$ of patients with NYHA FC IV receiving PAH-specific therapies, and $100 \%$ of patients with NYHA FC IV receiving supportive care

${ }^{\mathrm{d}}$ Epoprostenol was initiated in $50 \%$ of patients upon deterioration to FC IV in those receiving PAH-specific therapies and in $100 \%$ of patients receiving only supportive care

replications at willingness-to-pay values from Can $\$ 0$ to Can\$200,000 per QALY in both FC II and FC III PAH (Fig. 3). Tadalafil was the only other therapy, apart from supportive care, that had a greater than $0 \%$ chance of being cost effective; however, the probability for tadalafil ranged between only $20 \%$ and $30 \%$ of replications.

\section{Discussion}

We found sildenafil to be the most cost-effective treatment for first-line therapy in both patients with FC II and those with FC III PAH. The probabilistic sensitivity analysis identified considerable uncertainty surrounding the estimates of the incremental costs and QALYs associated with therapies, although there was little uncertainty relating to finding that sildenafil was the most cost-effective treatment, followed by tadalafil.

The uncertainty in the estimates was primarily due to uncertainty in the estimated effectiveness of treatments as opposed to the estimated costs. There were wide confidence intervals surrounding the estimates of improvement and worsening FC derived from the network meta-analysis. This stemmed not only from the limited number of welldesigned clinical trials that were available for inclusion within the analysis but also due to the substantial heterogeneity in design and patient population within the trials.
To address these issues, analysis focused on the patients who were naïve to $\mathrm{PAH}$ therapies and was conducted stratified by FC. The results were shown to be relatively insensitive to stratification by FC. Results were sensitive to assumptions regarding the proportion of patients initiating epoprostenol upon deteriorating to FC IV, and assumptions regarding the persistence of the $\mathrm{PAH}$ therapy effectiveness over the long-term. Even given this uncertainty, sildenafil was the most cost-effective therapy compared with other oral PAH therapies in all scenarios.

These results are not dissimilar to those of previously published models that focused on other jurisdictions; however, previous studies have been limited with respect to the PAH therapies under consideration, and examined only patients with FC III or IV disease.

In a previous cost-utility analysis of PAH treatments, Garin and colleagues found that sildenafil was the most cost-effective therapy from a US healthcare system perspective in patients with PAH FC III and IV [35]; this analysis did not include either riociguat or tadalafil within the possible treatment strategies. In their analysis, the transition probabilities for all drugs were based on adjusting the rate of improvement and worsening in FC with bosentan by the relative risk of improvement in the 6-min walk test compared with bosentan. Our study used a more robust approach of sourcing data from studies that had directly measured the rate of improvement and worsening 
Fig. 3 Cost-effectiveness acceptability curve by a PAH functional class II and b PAH functional class III at initiation of therapy. $Q A L Y$ qualityadjusted life-year, $P A H$ pulmonary arterial hypertension

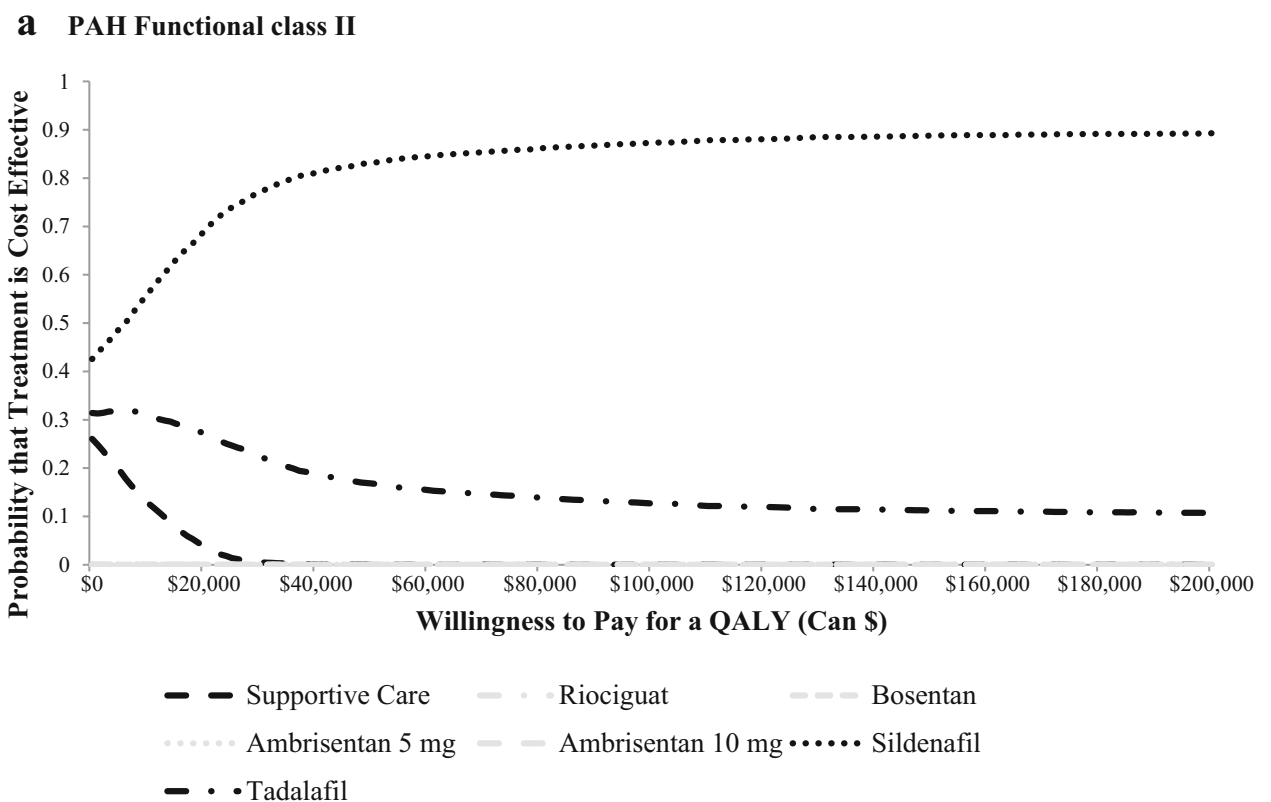

b PAH Functional class III

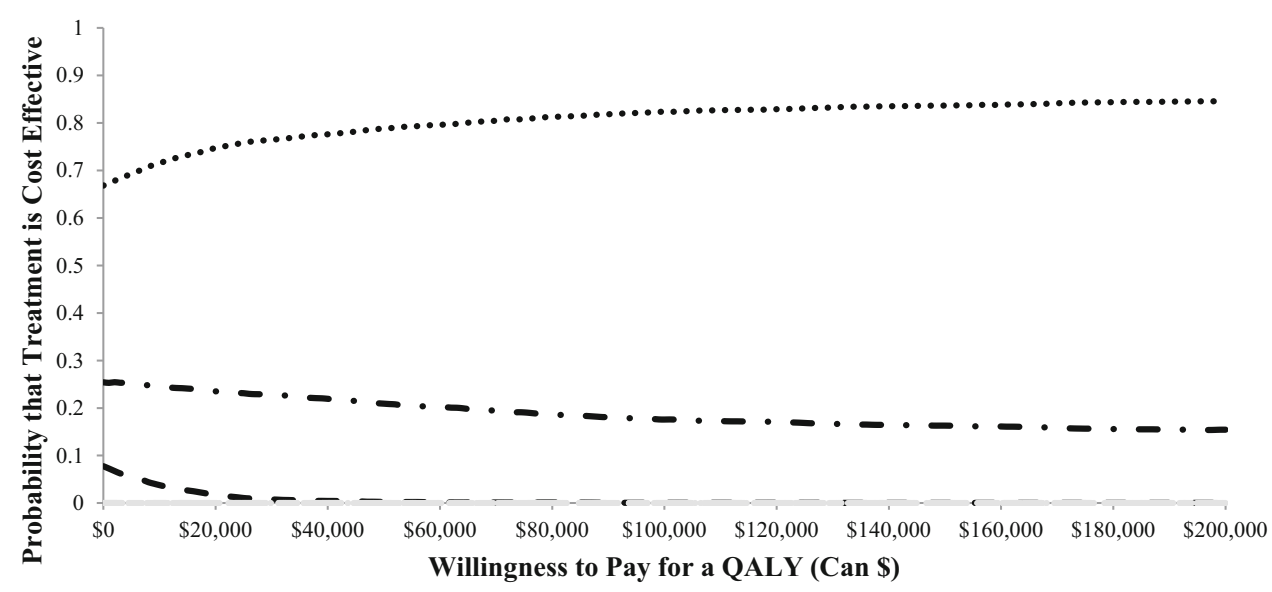

FC with each of the PAH therapies in patients naïve to PAH-specific treatment. Network meta-analysis provided a statistical approach to consolidate the information available from both head-to-head and placebo-controlled trials, allowing estimation of the comparative effects of treatments that had yet to be studied head-to-head [36].

In 2009, a UK study compared the cost effectiveness of PAH treatments, specifically epoprostenol, iloprost, sitaxentan, bosentan and sildenafil, individually versus supportive care in patients with FC III PAH [20]. Sildenafil dominated supportive care within this analysis, being both less costly and producing greater QALYs. When bosentan was compared with supportive care, the resulting ICER was $£ 27,000$ per QALY (2006 costs). This study differed from the current analysis in that it was not designed to allow a direct comparison of the cost effectiveness of PAH treatments relative to each other, but only relative to supportive care. Additionally, unlike the current study, which incorporated a survival benefit with treatment based on the treatment impact on FC worsening, the UK study derived a survival benefit from open-label follow-up studies [37]. There may be inherent bias in estimating long-term survival from this type of data as patients who enter into longterm study arms often differ significantly from the population included within randomised clinical trials [38, 39]. Given the lack of solid follow-up data, we felt the most 
appropriate way of incorporating a potential survival benefit was via directly measured effects on FC improvement and worsening.

The current analysis provides cost-effectiveness estimates stratified by initial FC for both class II and III. This stratified analysis is of particular interest given recent changes in reimbursement in some jurisdictions which provide differential coverage based on FC. The Ontario Drug Benefit program does not reimburse any PAH medication for patients with FC II disease but provides coverage for some PAH-specific medications for those with FC III disease, under its exceptional access program [40]. The results of the current analysis provide evidence against differential reimbursement between FC II and III.

We chose to use FC as the primary outcome for the estimation of gains in QALYs, with treatment based on the fact that FC has been established as a clinically relevant outcome in the treatment of PAH [5]. Although the 6-min walk test has often been the primary efficacy variable within PAH clinical trials, its relevance has been questioned in recent years due to its unclear relationship with clinically relevant outcomes such as hospitalizations, mortality, transplantation, or need for rescue therapy [41]. On the other hand, functional class has the benefit of not only having been shown to be associated with mortality but also with quality-of-life measures in PAH [23].

As with all modelling studies, the results are limited by the availability of data for populating the model. The reporting of results within clinical trials necessitated the assumption that the impact of treatment on improvement or worsening in FC overall could be applied regardless of the initial FC of the patient's disease. In the absence of more detailed reporting of results, and given that the majority of patients within the clinical trials were in FC II or III upon initiation of therapy, we consider this a valid and necessary assumption.

A second limitation of the analysis is that it included only PAH medication dosages that are therapeutically approved within Canada. In some cases, particularly with respect to sildenafil, there is evidence that doses used within clinical practice may be higher than the approved dose. However, the effectiveness data for the network meta-analysis was derived from studies with the approved doses and therefore this dose was used within the analysis.

Third, the model incorporated only the impact of adverse events associated with epoprostenol. This decision was based on evidence from meta-analyses which found that the rates of serious adverse events and discontinuations due to adverse events were comparable between oral PAH treatments and placebo or supportive care [42, 43]. Consequently, the omission of these adverse events should not affect the estimate of QALYs or costs, or bias the results.

The evidence for both the network meta-analysis and this economic evaluation could be strengthened through the conduct of well-designed head-to-head randomised controlled trials. Both were limited by the few studies available and the limited duration of the studies. Furthermore, the direct measurement of the impact of treatments on the quality of life in patients using a validated instrument, which may be used to estimate the QALY gain with treatment, would provide greater insight into the effectiveness of these therapies.

\section{Conclusion}

The results indicate that initiation of therapy with sildenafil in patients with FC II and III PAH would result in probable cost savings compared with other oral PAH therapies. This is an important finding as the costs associated with therapies for PAH are considerable. These findings also do not support differential funding of PAH therapies for patients with FC II versus FC III disease. It would seem appropriate, based not only on clinical trial evidence but also with respect to cost effectiveness, to initiate therapy with sildenafil, provided it is not contraindicated.

Acknowledgments The authors would like to thank Michel Boucher for his contributions to the analysis of the effectiveness data.

Author contributions Kathryn Coyle led the conception and design of the economic model, performed the analyses, interpreted the results, and composed the first version of the manuscript. Doug Coyle contributed to the design of the economic model and provided guidance regarding the analysis. Karen Lee and Julie Blouin contributed to the design of the study and assisted in interpretation of the results. Khai Tran, Mohammed Jabr, and Michel Boucher analysed the effectiveness data for incorporation within the model. Lisa Mielniczuk and John Swiston contributed to the design of the economic model and provided content expertise. All authors contributed to and revised drafts of the manuscript.

\section{Compliance with Ethical Standards}

Funding This research was supported by funds from the Canadian Agency for Drugs and Technologies in Health (CADTH).

Conflicts of interest Kathryn Coyle, Doug Coyle, Julie Blouin, Karen Lee, Mohammed F. Jabr, Khai Tran, and Mike Innes have no conflicts of interest. Lisa Mielniczuk has received research funds and honorariums, and has acted as a consultant for Actelion and Bayer. John Swiston has received honorariums and educational support from Actelion and Bayer; has received research funds from Actelion, Bayer, United Therapeutics, Eli Lilly, and GlaxoSmithKline; and has received payment for lectures from Bayer, GlaxoSmithKline, and Actelion.

Open Access This article is distributed under the terms of the Creative Commons Attribution-NonCommercial 4.0 International License (http://creativecommons.org/licenses/by-nc/4.0/), which permits any noncommercial use, distribution, and reproduction in any medium, provided you give appropriate credit to the original author(s) and the source, provide a link to the Creative Commons license, and indicate if changes were made. 


\section{References}

1. Humbert M, Sitbon O, Simonneau G. Treatment of pulmonary arterial hypertension. N Engl J Med. 2004;351(14):1425-36.

2. D'Alonzo GE, Barst RJ, Ayes SM, et al. Survival in patients with primary pulmonary hypertension. Results of a national prospective registry. Ann Intern Med. 1991;115:343-9.

3. McGoon MD, et al. Pulmonary arterial hypertension: epidemiology and registries. J Am Coll Cardiol. 2013;62(25 Suppl):D51-9.

4. Guillevin L, et al. Understanding the impact of pulmonary arterial hypertension on patients' and carers' lives. Eur Respir Rev. 2013;22(130):535-42.

5. Galie N, et al. Guidelines for the diagnosis and treatment of pulmonary hypertension: the Task Force for the Diagnosis and Treatment of Pulmonary Hypertension of the European Society of Cardiology (ESC) and the European Respiratory Society (ERS), endorsed by the International Society of Heart and Lung Transplantation (ISHLT). Eur Heart J. 2009;30(20):2493-537.

6. Benza RL, et al. The REVEAL Registry risk score calculator in patients newly diagnosed with pulmonary arterial hypertension. Chest. 2012;141(2):354-62.

7. McLaughlin VV. Survival in primary pulmonary hypertension: the impact of epoprostenol therapy. Circulation. 2002;106(12): 1477-82.

8. Sutton AJ, Abrams KR. Bayesian methods in meta-analysis and evidence synthesis. Stat Methods Med Res. 2001;10(4):277-303.

9. Canadian Agency for Drugs and Technologies in Health. CADTH therapeutic review. Drugs for pulmonary arterial hypertension: comparative efficacy, safety, and cost-effectiveness. 16 Mar 2015. https://www.cadth.ca/drugs-pulmonary-arterial-hypertension. Accessed 8 April 2015.

10. Canadian Agency for Drugs and Technologies in Health. Guidelines for the economic evaluation of health technologies. Ottawa (ON): Canadian Agency for Drugs and Technologies in Health; 2006.

11. Peacock AJ, et al. An epidemiological study of pulmonary arterial hypertension. Eur Respir J. 2007;30(1):104-9.

12. Statistics Canada. Life tables, Canada, provinces and territories, 2009 to 2011. Statistics Canada: catalogue no. 84-537-X-No.003. 2009. http://www.statcan.gc.ca/pub/84-537-x/84-537-x2013005eng.htm.

13. Thenappan T, et al. Survival in pulmonary arterial hypertension: a reappraisal of the NIH risk stratification equation. Eur Respir J. 2010;35(5):1079-87.

14. Fleurence RL, Hollenbeak CS. Rates and probabilities in economic modelling: transformation, translation and appropriate application. Pharmacoeconomics. 2007;25(1):3-6.

15. Ling Y, et al. Changing demographics, epidemiology, and survival of incident pulmonary arterial hypertension: results from the pulmonary hypertension registry of the United Kingdom and Ireland. Am J Respir Crit Care Med. 2012;186(8):790-6.

16. Galie $\mathrm{N}$, et al. Ambrisentan for the treatment of pulmonary arterial hypertension: results of the ambrisentan in pulmonary arterial hypertension, randomized, double-blind, placebo-controlled, multicenter, efficacy (ARIES) study 1 and 2. Circulation. 2008;117(23):3010-9.

17. Channick RN, et al. Effects of the dual endothelin-receptor antagonist bosentan in patients with pulmonary hypertension: a randomised placebo-controlled study. Lancet. 2001;358(9288): 1119-23.

18. Galie N, et al. Sildenafil citrate therapy for pulmonary arterial hypertension. N Engl J Med. 2005;353(20):2148-57.

19. Ghofrani HA, et al. Riociguat for the treatment of pulmonary arterial hypertension. N Engl J Med. 2013;369(4):330-40.
20. Chen YF, et al. Clinical and cost-effectiveness of epoprostenol, iloprost, bosentan, sitaxentan and sildenafil for pulmonary arterial hypertension within their licensed indications: a systematic review and economic evaluation. Health Technol Assess. 2009;13(49):1-320.

21. Galie N, et al. A meta-analysis of randomized controlled trials in pulmonary arterial hypertension. Eur Heart J. 2009;30(4):394-403.

22. Macchia A, et al. A meta-analysis of trials of pulmonary hypertension: a clinical condition looking for drugs and research methodology. Am Heart J. 2007;153(6):1037-47.

23. Keogh AM, et al. Quality of life in pulmonary arterial hypertension: improvement and maintenance with bosentan. J Heart Lung Transplant. 2007;26(2):181-7.

24. Drabinski A, Williams G, Formica C. Observational evaluation of health state utilities among a cohort of sepsis patients. Value Health. 2001;4:130.

25. Einarson TR, Granton JT, Vicente C, et al. Cost-effectiveness of treprostinil versus epoprostenol in patients with pulmonary arterial hypertension: a Canadian analysis. Can Respir J. 2005; 12(8):419-25.

26. Government of Saskatchewan. Drug Plan and Extended Benefits Branch. Saskatchewan Online Drug Formulary. 2014 [cited 4 Sep 2014]. http://formulary.drugplan.health.gov.sk.ca. Accessed 10 April 2014.

27. Badesch DB, et al. Pulmonary arterial hypertension: baseline characteristics from the REVEAL Registry. Chest. 2010;137(2):376-87.

28. Ontario Council on Community Intepreting. Ontario case costing initiative. 2013 [cited 4 Sep 2014]. http://hsimi.on.ca/hdportal/.

29. Ontario Ministry of Health and Long Term Care. Schedule of benefits for physician services under the Health Insurance Act. 2014. http://www.health.gov.on.ca/english/providers/program/ ohip/sob/physserv/physserv_mn.html. Accessed Jul 2014.

30. Ontario Ministry of Health and Long Term Care. Ontario Health Insurance (OHIP) schedule of benefits and fees. Schedule of benefits for laboratory services. 2014. http://www.health.gov.on. ca/english/providers/program/ohip/sob/lab/lab_mn.html. Accessed Jul 2014.

31. Letarte $\mathbf{J}$, et al. Patient characteristics and costs of severe sepsis and septic shock in Quebec. J Crit Care. 2002;17(1):39-49.

32. Bank of Canada. Bank of Canada inflation calculator. 2014. http://www.bankofcanada.ca/rates/related/inflation-calculator/. Accessed 10 April 2014.

33. Roman A, et al. Cost effectiveness of prostacyclins in pulmonary arterial hypertension. Appl Health Econ Health Policy. 2012;10(3):175-88.

34. Briggs AH, Sculpher MJ, Claxton K. Decision modelling for health economic evaluation, vol. 1. Oxford: Oxford University Press; 2006.

35. Garin MC, et al. Cost-utility of treatments for pulmonary arterial hypertension: a Markov state-transition decision analysis model. Clin Drug Investig. 2009;29(10):635-46.

36. Mills EJ, Thorlund K, Ioannidis JPA. Demystifying trial networks and network meta-analysis. BMJ. 2013;346:f2914.

37. Sitbon O, et al. Survival in patients with class III idiopathic pulmonary arterial hypertension treated with first line oral bosentan compared with an historical cohort of patients started on intravenous epoprostenol. Thorax. 2005;60(12):1025-30.

38. Pocock SJ, Elbourne DR. Randomized trials or observational tribulations? N Engl J Med. 2000;342(25):1907-9.

39. Taylor GJ, Wainwright P. Open label extension studies: research or marketing? BMJ. 2005;331(7516):572-4.

40. Ontario Ministry of Health and Long Term Care. Ontario Ministry of Health and Long Term Care Exceptional Access Program: EAP reimbursement criteria for frequently requested drugs. 2014. http://www.health.gov.on.ca/en/pro/programs/drugs/pdf/frequently_ requested_drugs.pdf. Accessed 6 Feb 2015. 
41. Savarese G, et al. Do changes of 6-minute walk distance predict clinical events in patients with pulmonary arterial hypertension? A meta-analysis of 22 randomized trials. J Am Coll Cardiol. 2012;60(13):1192-201.

42. He B, et al. Meta-analysis of randomized controlled trials on treatment of pulmonary arterial hypertension. Circ J. 2010;74(7):1458-64.

43. Zheng YG, et al. Oral targeted therapies in the treatment of pulmonary arterial hypertension: a meta-analysis of clinical trials. Pulm Pharmacol Ther. 2014;29(2):241-9.

44. Ontario Ministry of Health and Long Term Care. Ontario Drug Benefit Formulary/Comparative Drug Index. 29 Jun 2015; 42. http://health.gov.on.ca/en/pro/programs/drugs/formulary42/edition_ 42.pdf. Accessed 7 April 2014.

45. Government of Ontario. Home oxygen therapy policy and administration manual. Assistive Devices Program, Ministry of Health and Long-Term Care. Toronto (ON): Government of Ontario; 2014.

46. Office of the Auditor General of Ontario. 2010 Annual Report. 2014. http://www.auditor.on.ca/en/reports_2010_en.htm. Accessed 20 April 2014. 